\title{
LO COTIDIANO: UNA FORMA BÁSICA DE MIRAR LA DIVERSIDAD
}

Dora Maria Hernández Vargas ${ }^{1}$

No pido eternidades Llenas de estrellas blancas.

Pido ternura, cena, Silencio, pan y casa... Jorge Debravo

\section{Resumen}

A partir de una estrofa de un poema de Jorge Debravo, la autora de este ensayo lleva al lector a reflexionar sobre la manera en que el respeto y la valoración de la diversidad presente en todo ser y hacer humano, deben abordarse desde lo cotidiano, desde esa necesidad diaria de temura, cena, silencio, pan y casa.

Nuestro célebre poeta hace una de las afirmaciones más consistentes y enérgicas cuando dice, con gran belleza poética y como si toda la humanidad dijera con él: "Soy hombre, he nacido, tengo piel y esperanza...". Debravo logra expresar e identificar algunas de las necesidades y derechos básicos de las personas en este breve poema, con una fuerza impresionante. La autora considera importante que, la persona no pide "etemidades llenas de estrellas blancas". Y concuerda con el poeta cuando explica suavemente: "Pido temura, cena, silencio, pan y casa...".

\section{Palabras claves}

Diversidad, respeto trabajo, ser humano.

\section{Abstract}

I do not ask for eternities Full of white stars I ask for tenderness, supper, Silence, bread and home... Jorge Debravo

In the following pages, using a fragment, the author takes the reader into a journey of thinking about the significance of how respect for human diversity must be approached on a daily

1 Máster en Pedagogía con énfasis en Diversidad en los Procesos Educativos, Universidad Nacional y Licenciada en Ciencias de la Educación con Énfasis en Educación Preescolar, también de la Universidad Nacional. Es académica de la División de Educación Básica del CIDE-UNA. Forma parte del equipo de investigación del proyecto "El reconocimiento de las identidades educativas en la construcción del proyecto de escuela". 
basis, since a regular need for tenderness, supper, silence, bread and home.

Our well-known poet makes one of the most consistent and vigorous statements that I can think of when he writes with poetic beauty, like if all humankind would say with him: "I am a man, I was born, I have skin and hope...". Debravo manages to express and identify remarkably powerfully in this brief poem, some of the basic people's rights and needs. In what I consider the major fragment of his poem he says: "I do not ask for eternities full of white stars". Then, he gently explains: "I ask for tenderness, supper, silence, bread and home...".

\section{Key Words}

Diversity, respect, worth, human being.

\section{TERNURA: una necesidad básica}

i pensamos en el acto educativo, pensamos en el acto cotidiano. Más $\checkmark$ adelante abordaré con mayor profundidad este punto. Por ahora, cabe decir que desde la cotidianeidad, es posible construir lo pedagógico con significancia. Y cuando algo resulta significante para alguien, es cuando ese algo logra entablar un vínculo con la emotividad, con los afectos y los deseos de una persona. No se trata sólo de comprensión, de análisis o de descubrimiento. No se trata tan sólo del acercamiento que tiene el sujeto con el objeto, de la manera como lo propone el método científico. El significado afiora cuando existen asociaciones con elementos internos igualmente plenos de significado y a los que les atribuimos valor. Sucede así, porque somos capaces de sentir, de apreciar, de valorar, de amar. Esto nos identifica como seres humanos, quizá más, pienso yo, que nuestras capacidades para razonar, entender o argumentar, tan ensalzadas en nuestra época. Ambas posibilidades no debieran aislarse ni proponerse como antagónicas; por el contrario, ha de enriquecerse y complementarse. Esto nos pone en el camino de desarrollar nuestra plenitud como personas.

Ahora bien, la práctica escolar actual aparenta no tener lugar para la ternura. Efectivamente, desde que la educación de los menores de edad fue expropiada por el Estado, despojando a la familia, la ternura ha sido relegada a la marginalidad. Algunas veces, parece que con la estructuración de las instituciones escolares, la educación se ha burocratizado y se ha tornado fría e impersonal. De acuerdo con el modelo burocrático de educación, el vínculo entre los educandos y el educador se manifiesta esencialmente dentro del ámbito de lo racional. En el peor de los casos, el vínculo tiene un predominio 
administrativo. Como afirma Restrepo (1998), "la razón burocrática proyecta en el individuo las causas del desencuentro, resistiéndose a echar una mirada sobre su propio funcionamiento. Al negar la importancia de las cogniciones afectivas, la educación se afirma en una pedantería del saber que se mantiene subsidiaria de una concepción de razón universal y apática, distante de los sentimientos y los afectos, afianzadora de un interés imperial que desconoce la importancia de ligarse a contextos y seres singulares" (p. 51).

Resulta interesante considerar esto a la luz de la comprensión de que la educación no es neutral, porque no podemos "desconectarla" de toda tesis, digamos aquí, relativa al ser humano como sujeto (u "objeto") de la enseñanza y aprendizaje; de manera que todo supuesto y afirmación de implicaciones pedagógicas no pueden considerarse neutrales.

Así como los actos humanos no son neutrales, en cuanto no son mecánicos (y aún los que pueden considerarse mecánicos tienen una razón de ser) y el ser humano es sujeto de voluntad y de personalidad (tiene definiciones constitutivas propias), la educación sólo es plena en el respeto por esa diversidad. En tanto que la educación no respete y valore dicha diversidad, se convierte en un mecanismo de conservación del poder (considerando este como fin último, aunque sea oculto), como dice Restrepo, afianzadora de un interés imperial, que ya no cumple su función en el desarrollo de las potencialidades humanas, ricas en diversidad y expresiones.

La educación ha de considerar al ser humano como sujeto o como objeto y en esta consideración se destaca su no neutralidad. La ternura que se vive como expresión del respeto por el sujeto diverso da cuenta de su opción por la persona. La violencia que se manifiesta como espejo de la negación del sujeto, convirtiéndolo en objeto, destaca su opción por el poder. En este caso, no existe correspondencia ni diálogo, sino dominación.

Es así que la ternura se erige como una necesidad básica en el contexto educativo, en tanto que humano, pues ha de entenderse como "la manifestación afectiva más concreta y englobante del amor humano" (Rovira en López y España, 1998, p. 254). La ternura como imperativo en las relaciones entre las personas es una alternativa frente a la violencia, la indiferencia, el abuso y la falta de diálogo. También debe ser vista como clima y cualidad de aprendizaje, como gesto que integra diversidades; la diversidad que es el otro. La ternura tiene que ser como acogida y bienvenida.

En el espectro de necesidades que demandan del ser humano su satisfacción, la ternura se revela como pan del alma, como manantial al sediento. Su exigencia es tal, que en su ausencia la propia valía sufre, la capacidad de 
relacionarse languidece, el deseo por la vida se ve debilitado, la habilidad para la esperanza falla.

Rovira, en López y España (1998), señala: "vivimos en una cotidianeidad falta de sueños, mecanicista, robotizada. Todos los días parecen iguales, no hay tiempo para lo pequeño, para lo profundo" (p. 252). La Escuela asume frecuentemente el acto educativo como aspiración de conductas controladas, de conocimientos estáticos, de reproducción simétrica, de palabra medida, en un ambiente donde la expresión creativa y personal (diversa) no encuentra forma de manifestarse. El currículo está desprovisto usualmente de la ternura de concebir a cada uno como único y única, de acercarse a él o ella desde su existencia. Es posible más bien encontrar la violencia en la exaltación del contenido, como fin del proceso. La violencia dibujada en el estándar de rendimiento académico a quien cada cual debe acceder como pueda, es la negación de las necesidades, las características y las vivencias del otro. La violencia lleva a la arbitrariedad y el abuso del poder. La autoridad pasa de ser un acto de ternura y servicio, a favor del desarrollo del semejante y su potenciación, y se convierte en un espacio de alienación.

En el marco de una verdadera consideración de la diversidad humana dentro del acto educativo, yace la disposición a la ternura; aquella que reconoce al otro, no como quisiéramos que fuera, sino como en realidad es. La ternura busca los caminos para llegar a él y ella con ese conocimiento, con esa enseñanza y aprendizaje; ofrece multiplicidad de recursos, ambientes y tiempos, como el que recuerda que la vida tiene colores; se enfrenta a personas en sus contextos propios, sin violentar su identidad. La ternura confiere a un tú, el mismo valor de un yo, porque dignifica al ser humano y lo concibe como fin, en tanto que la educación se convierte en un medio.

El "tengo piel y esperanza" de Debravo, nos remite a esa constitución del ser que reclama ser acariciado y confortado, reconocido y celebrado. El acto pedagógico requiere acercamiento (no sólo con respecto al contenido) mediante la ternura. La pedagogía (paideia) debe concebirse no como la suma de nociones adquiridas, sino como la formación total del hombre y la mujer como miembro de un ambiente social. En este sentido, resulta valiosa la advertencia que nos hace Rovira en López y España (1998):

"A nuestro presente, en el que el pragmatismo y el tecnicismo se han apoderado de la mayoría de nuestras relaciones, sentimientos y pensamientos, es menester recordarle la parte humana del trabajo humano de vivir, pues; somos 'modelo' para los jóvenes y las jóvenes, y los 
niños y las niñas que comparten con nosotros (as) este espacio y este tiempo, y para serlo de una manera a escala humana es preciso humedecer nuestra persona y nuestras relaciones. (Lo tierno es húmedo, lo tierno es flexible, lo tierno es posibilidad)" (p. 259).

\section{CENA: hacia una pedagogía dialógica}

La cotidianeidad del diálogo es también un imperativo en la construcción de pedagogías que consideran y valoran la diversidad en el acto educativo. Es como la cena de cada día, que abre el espacio común de conversación familiar y entre amigos.

Núñez, en López y España (1998), destaca que una pedagogía de la comunicación o pedagogía dialógica,

"(...) lo que sostiene es que conocemos y nos comprometemos con la realidad para cambiarla a partir de un protagonismo dado por todos, en el conjunto de los conocimientos y experiencias vividas. Una base práctica del conocimiento científico, crítico y transformador que se busca aqui, consiste en que nada puede caminar en derechos humanos sin que se tome en cuenta al otro muy seriamente, muy esencialmente. Es un punto de partida democrático en el cual se da a otros 'la debida consideración' (Gamboa, 1990). La fuente de ese conocimiento no es otra que la cotidianidad autónoma de cada uno y de todos. Su criterio de verdad es el diálogo esencial, aquel que puede cambiarnos por dentro y por fuera" (pp. 432-433) (lo resaltado no es del texto original).

Como dice este autor, es un punto de partida democrático, desde la debida consideración al otro. Es un reconocimiento del pluralismo social y un acercamiento a la convivencia justa y pacífica. Es aceptar, desde la misma ternura de que hablamos, que el otro, esencialmente diverso, tiene igual derecho que yo a expresarse, a participar, a construir, pero no desde la individualidad y el aislamiento, sino desde la comunidad. Respetar y valorar la diversidad humana no se convierte así, en la validación del individualismo y el egoísmo, sino más bien en la conjunción de esas diferencias o diversidades en tomo a la búsqueda del bien común. Así, el diálogo es esencial, y se erige como la piedra angular, necesariamente soportante de todo esfuerzo por construir una sociedad más justa, solidaria y respetuosa del derecho y bien de todos. 
Desde este punto de vista, la democracia no sólo resulta el punto de partida, sino, en cierta forma, el punto de llegada. El punto de llegada en cuanto aspira a un tipo de convivencia que supone la participación en y desde el respeto por la diversidad humana. Pero cuidado, ella debe "combatir el poder absoluto, el del despotismo militar y el del partido totalitario, pero también debe poner límites a un individualismo extremo que podría divorciar por completo la sociedad civil y la sociedad política para dejar librada esta última, ya a los juegos fácilmente comuptores, ya al poder invasor de las administraciones y las empresas" (Touraine, 1994, p. 320). Esto quiere decir, no sólo que por medio de la democracia es posible una participación de los diferentes sectores sociales, sino que mediante su acción vigorosa se evitarán los excesos y los abusos.

Enseñar a los niños y las niñas dentro del marco de la democracia y para una vida en democracia es un reto muy urgente. Rojas, en López y España (1998), convoca:

“... a los sectores educativos, para que asuman un papel protagónico en el debate de los aspectos fundamentales del régimen democrático en una forma interdisciplinaria, desde la búsqueda de soluciones a los ingentes problemas que afrontan nuestras sociedades. Como se ejemplifica frecuentemente -la democracia es igual a un músculo, si no se ejercita se atrofia.- El debate debe ser un aspecto importante en la comprensión de las problemáticas que enfrentan cotidianamente los sectores más amplios de nuestra América" (p. 164) (lo resaltado no es del texto original).

El debate supone una concepción acertada de la diversidad humana, a partir del hecho de que, a pesar de que cada sujeto es diferente con respecto a los demás (así como sus ideas), es posible el acuerdo. Quisiera referirme a este supuesto desde algunos aspectos de la propuesta de J. Habermas.

Habermas cree en la posibilidad de hacer aparecer lo universal en la comunicación entre las experiencias particulares nutridas por la particularidad de un mundo vivido ("Lebenswelt"), de una cultura. Según este autor, uno no debe contentarse con los compromisos que ofrece la política liberal, ni con una tolerancia que yuxtapone los particularismos en lugar de integrarlos. Para él, es necesario admitir que no hay democracia sin ciudadanía y que no hay ciudadanía sin acuerdo, no sólo sobre procedimientos e instituciones, sino también sobre contenidos. 
Pero, ¿cómo vincular lo universal con lo particular? Habermas considera que mediante la comunicación y, más concretamente, a través de la discusión y la argumentación, que permiten reconocer en el otro lo que es más auténtico y lo que se refiere a un valor moral o a una norma social universalista. Esta disposición de respetar y escuchar al otro se manifiesta como un fundamento más sólido de la democracia que el enfrentamiento de intereses que conduce a compromisos y a garantías jurídicas.

Habermas se une a numerosos teorizadores para quienes una sociedad no es sólo un conjunto de producción, sino también una colectividad que tiene exigencias de integración social y conservación de sus valores culturales; en términos más concretos, "ese conjunto es aquel en el que la educación y la justicia son tan importantes como la economía y la política" (Touraine, 1994, p. 331).

Desde su punto de vista, la diversidad humana (y por ende, de pensamiento) es supuesto fundamental, digno, válido y necesario para la vida social. Se trata de una propuesta que trasciende, como el mismo Habermas señala, el mero enfrentamiento de intereses para procurar un encuentro con el otro a través del diálogo atento, racional y comprometido. La convivencia así vista, no sólo supone la interacción desde la diversidad de los sujetos, a quienes más bien debiéramos llamarles ciudadanos desde su propuesta, sino que supone la integración. En este sentido, el acuerdo adquiere el valor de fruto de procesos dialógicos profundos y comprometidos. No se trata sólo de la conversación respetuosa, la exposición de puntos de vista o la expresión de necesidades, intereses o perspectivas; ni tampoco de conocer lo que el otro piensa de manera superficial y ajena.

El tipo de diálogo que propone Habermas exige disposiciones y habilidades importantes en torno, no sólo a la capacidad de argumentación y discusión, sino a la capacidad de encontrarse con el otro en un acto de acogida como es la escucha, donde ambas partes pretenden lograr la integración social "y la conservación de sus valores culturales”, como líneas arriba se apuntó.

El diálogo, así enfocado, adquiere un sentido dinámico y profundo y se convierte en la habilidad necesaria para la integración social real y para la convivencia. La con-vivencia que se traduce en la vivencia com-partida, donde no se trata sólo de respetar (en el sentido de tolerar) al semejante, sino de saberme parte de su mundo. Precisamente, la carencia de esta habilidad se ilustra con fuerza viva en una de las problemáticas que nos exigen el más alto reto de diálogo: la contaminación global. Esta problemática refleja la incapacidad que actualmente demuestran muchas sociedades para comprender la convivencia. 
Sólo se manifiesta una conciencia del interés individual y egoísta y un impresionante abuso del poder.

El diálogo, cuyo sentido etimológico proviene de día (a través de) y legein (hablar), se constituye en una necesidad humana, no sólo para la resolución de conflictos o para el acercamiento como sociedad, sino para la verdadera con-vivencia democrática; porque como dice Touraine (1994): "La democracia no es el triunfo del pueblo; es la subordinación del mundo de las obras, de las técnicas y de las instituciones a la capacidad creadora y transformadora de los individuos y las colectividades" (p. 344).

Ahora bien, si pensamos en el reto que tenemos en la construcción de este tipo de sociedad democrática, nos damos cuenta que la educación es una de las más eficaces herramientas en la consecución de estos fines. En primer lugar, porque la educación tiene naturaleza política. Freire, en su obra La naturaleza política de la educación, establece lo siguiente:

"En primer lugar, la educación, sea en la universidad, la escuela secundaria, la primaria o en la alfabetización de adultos, es un acto político (...) Un acto educativo tiene naturaleza política y un acto político tiene naturaleza educativa. Si esto es habitualmente así, sería erróneo decir que sólo la educación de Latinoamérica tiene naturaleza política. La educación tiene naturaleza política en todo el mundo. En términos metafisicos, la política es el alma de la educación, su mismo ser, sea en el primer mundo o en el tercer mundo (...)

En segundo lugar, ya sea en Brasil o en cualquier otro sitio, ya se trabaje en alfabetización o en estudios secundarios, la educación consiste en una serie de teorías llevadas a la práctica. No podemos evadir ese hecho (...)

Lo que debemos definir es lo referente a ¿cuáles son nuestras posiciones respecto del conocimiento?, ¿cuáles son nuestras concepciones en la teoría del conocimiento?, ¿cómo abordamos el objeto de conocimiento?, ¿lo poseemos?, ¿lo llevamos en el portafolios para distribuirlo entre nuestros estudiantes?, ¿utilizamos esie objeto de conocimiento para alimentar a los estudiantes o para estimularlos a conocer?, ¿los estimulamos a asumir el rol de sujetos, o el de receptores pasivos de nuestro conocimiento?" (1990, pp. 184-185).

Este hecho encuentra relación con la afirmación hecha líneas arriba de que la educación no es neutral. Existe una línea entre lo pedagógico y lo 
político, entre la acción concreta y la acción estructural. Esa acción concreta se refiere al carácter cotidiano de la acción pedagógica, mientras que la acción estructural tiene relación con el ámbito donde lo político cumple un papel importante. Pero la educación no está desligada de ninguno de los dos. Ejerce su poder en ambos sentidos, que se encuentran interrelacionados en el acto pedagógico.

Como afirma categóricamente Núñez, en López y España (1998), "Cualquier proceso educativo que se considere tal desde un enfoque del respeto a la persona humana luchará incansablemente por engranar dos niveles: lo cotidiano y lo estructural" (p. 432) (lo destacado no es del texto original). Así que esta naturaleza política de la educación encuentra su expresión en la cotidianeidad, en la vivencia y en la con-vivencia que ella implica. Optamos cotidianamente por una forma de abordar la diversidad y de valorar a la persona (como sujeto o como objeto), con implicaciones profundamente políticas.

En segundo lugar, la educación es una herramienta para construir una sociedad democrática a través del diálogo, porque es formadora de cultura. Transforma, en primer lugar, las estructuras personales y, por lo tanto, las estructuras sociales. En efecto, muchas de las estructuras de las instituciones sociales están conformadas por su manera de pensar, de ver el mundo, de asumir las relaciones, de vivir. En este sentido, podemos decir que representar y manifiestan la cultura de un grupo humano. Por ejemplo, pienso que la existencia de la burocracia es posible porque existe, como parte de la cultura, una forma de pensar, de reaccionar, de sentir, de enfrentarse, de relacionarse, de decidir que caracteriza a un determinado grupo social; de forma que aunque la legislación y las estructuras externas cambiaran, probablemente la institución realmente no cambiaría significativamente.

Por eso, el proceso de transformación social debe ser un proceso de transformación de la cultura. Se trata de algo así como la cuestión de echar vino nuevo en odres viejos. Es necesario renovar ambas cosas: el aspecto personal o cultural, humano y el aspecto estructural, o sea, los mecanismos necesarios para que una nueva forma de funcionar, relacionarse y vivir sean posibles. En este sentido, podemos decir que la educación tiene en sus manos una importante tarea de transformación cultural y social. Aclaro que cultural en tanto persigue la consecución de intenciones edzicativas cimentadas en un compromiso con la dignidad del crecimiento y la formación integral y total humana (la persona como sujeto y como fin), que se establece como proyecto social a veces contrario a ciertas manifestaciones culturales que van en detrimento de su misión. 
En esta integración de lo estructural y lo cotidiano, yace en gran parte la fuerza transformadora del proyecto educativo. Un análisis de las prácticas pedagógicas cotidianas se hace necesario en la búsqueda por evaluar en qué medida lo estructural está sufriendo también transformaciones. Como interrogaba Freire, ¿utilizamos este objeto de conocimiento para alimentar a los estudiantes o para estimularlos a conocer?, ¿los estimulamos a asumir el rol de sujetos, o el de receptores pasivos de nuestro conocimiento?

Estas prácticas se refieren a la vivencia cotidiana, reflejo de las estructuras sociales. Se hace necesaria una reflexión, por ejemplo, sobre las reglas que articulan las relaciones jerárquicas en la escuela; así como los criterios que en ella se manifiestan cotidianamente, con el fin de determinar si se dan en forma implícita o explícita, si colaboran con el ảesarrollo de la autonomía o si más bien lo dificultan, si promueven o no la comprensión crítica del papel de la regla y la norma. Sería importante también reflexionar si dichas prácticas y usos de reglas de criterios, que procuran mediar la convivencia escolar, generan una desconfianza significativa con respecto a las instituciones sociales, que pueden ser leídas como un poder central, abstracto, que irrumpe arbitrariamente para romper con la regularidad y el ordenamiento escolar.

A la luz de estas consideraciones, es posible reconocer, por una lado, a la escuela como una institución que participa en forma sustantiva en la construcción de la ciudadanía y, por el otro, que se hace necesario estudiar de manera sistemática sus formas de participación en este proceso, si es que deseamos educar para la democracia en un proceso más consciente y reflexionado (Elizondo, 2000).

\section{SILENCIO: hacia una pedagogía de la comunicación}

Considero el silencio parte del proceso de comunicación. La palabra compartida se construye sobre el silencio del otro. La verdadera comunicación tiene como base tiempos de hablar y tiempos de callar. Una pedagogía que considera la diversidad de las personas como una práctica cotidiana es una pedagogía de la comunicación. Es necesario este tipo de pedagogías que acojan lo diferente, lo múltiple y lo complejo, y que lo hagan desde los niños y niñas que aprenden a celebrar su vida, junto a los demás, mientras disienten, cuestionan, critican, abren brechas con sus propuestas. La comunicación va más allá de las palabras. 


\section{PAN: el valor de lo cotidiano}

Decíamos líneas arriba, que cualquier proceso educativo que se considere tal desde un enfoque del respeto a la persona humana, luchará incansablemente por engranar dos niveles: lo cotidiano y lo estructural (Núñez, en López y España, 1998). Es en lo cotidiano donde la educación toma forma. La educación, entendida como formación total e integral de la persona, no es posible en un corto tiempo, pues requiere toda la vida. Es desde lo cotidiano que es posible aprender, verdaderamente. Sobre todo desde pedagogías como las que proponemos, donde el aprendizaje de habilidades para la ternura, el diálogo y la comunicación se hace imperativo.

Lo cotidiano conforma cada uno de los hilos de la existencia. Si lo vemos independientemente, es un día, si lo vemos en su totalidad, es toda una vida. Así, el acto pedagógico es el acto de tejer la intrincada trama de la vida y de la con-vivencia humana, soñando cómo queremos que luzca, planificando cuál hilo va primero y cuál después, para darle mayor belleza al conjunto, proveyendo de los mejores materiales para que sea consistente y caluroso. Si tejemos, descuidadamente, de manera irreflexiva, como si el valor de cada hilo careciera de significado, al final obtendremos un paño informe, frágil y falto de toda belleza y sentido de obra.

Cada palabra tiene sentido por sí misma, como cada día guarda un hermoso significado. Pero al unirlas, podemos expresar más profundas e intrincadas frases con un mayor grado los alcances del alma. Así resulta lo cotidiano. Dentro del acto pedagógico, cada día tiene su sentido y su valor; sin embargo, uniendo cotidianeidades, es posible contemplar la belleza de la transformación humana. Ojalá en personas más tiernas, capaces de con-vivir.

El respeto y la valoración de la diversidad presente en todo ser y hacer humano, deben abordarse desde lo cotidiano, como hemos estado ensayando, desde esa necesidad diaria de ternura, cena, silencio, pan y casa. El pan que "com-partimos" cada día resurge como emblema de la necesidad que tenemos como seres humanos de ser sustentados cada día. No somos seres de "una vez y para siempre", sino seres dotados de necesidades que deben ser suplidas diariamente. La necesidad de ser tratados y estimados desde nuestra propia diversidad, es la necesidad y el derecho de cada día. 
Una casa es un lugar para vivir. Pero es más que eso. Significa; tiene vínculos con los más profundos anhelos en el corazón del ser humano; es un espacio para la intimidad, para la ternura, para el diálogo, para la comunicación, para la "con-vivencia", para el aprendizaje... para ser uno mismo.

Esta vivencia cotidiana de la diversidad nos invita a tenerla como nuestra casa. Una casa para todos. La necesidad de una educación que respeta y parte de la diversidad de cada ser humano, logra responder a la más profunda necesidad de todo niño y niña, de todo hombre y mujer, como decíamos: ser conocido y aceptado tal cual es.

En la medida en que, como escuela, proveamos un ambiente y un tipo de relaciones donde las personas puedan ser quienes son y no necesiten asumir roles funcionales dentro de un sistema cerrado de reglas y expectativas, esa escuela sería realmente para la diversidad y estaría cumpliendo el papel que le corresponde en las vidas de las personas: el de estar al servicio de su desarrollo y realización. Esto considerando que, como se ha dicho, a veces parece ser que los individuos están al servicio de la escuela, que tiene sus propios fines y busca su propia legitimación. Así, el fin llega a ser ella misma como sistema, como institución, como emblema, que promete orden, estabilidad, superación o seguridad social.

Nótese que hablamos de una escuela para la diversidad, porque creemos que desde una propuesta así, se debe partir del reconocimiento de las personas como valiosas y aceptables (con ternura). No sólo todos sus actores se desenvuelven en medio de la diversidad, no sólo es tomada en cuenta, no sólo parte de ella, sino que está al servicio de personas diversas, es para ellas en sus particularidades. Pero no se queda allí. Reconoce a cada persona; reconoce que nos definimos y conformamos en relación con otros. Y aún más, reconoce la diversidad como parte valiosa y necesaria de las interacciones humanas.

Hablamos de una escuela que no sólo es como una casa, sino como un hogar; pues ha dejado de ser el cascarón y la estructura de algo que ya no está allí. Nos referimos a una escuela que se comunica y que intenta tocar no sólo la mente, sino el corazón; ese lugar misterioso donde yacen los afectos, las motivaciones, las pasiones, lugar de nuestras alegrías y de nuestros más profundos recuerdos; fuente de nuestras vidas y nuestros sueños.

Es una escuela que alcanza no sólo la mente, sino el corazón; porque al hablar de diversidad no sólo hablamos de las diferencias en estilos de 
aprendizaje que tienen los niños y las niñas entre sí; de las diferencias en sus capacidades físicas, mentales o emocionales; de las diferencias debidas a procedencias étnicas, de clase social o de idioma; de las diferencias de género o religión. Nos referimos a una concepción de ser humano y de educación que va más allá de la consideración de las diferencias y se centra en satisfacer necesidades humanas, como la de ternura, cena, silencio, pan y casa. 


\section{REFERENCIAS}

Elizondo, A. (2000). Cómo van conformando los niños y las niñas prácticas pedagógicas civicas en el quehacer cotidiano de la escuela. Investigación realizada en la Universidad Pedagógica Nacional de México. Disponible en línea: http://www.azc.uam.mx

Freire, P. (1990). La naturaleza política de la educación. Barcelona: Paidós.

López, R. y España, O. (comp.) (1998). Cultura, identidades y ciberespacio: III Congreso Latinoamericano de Humanidades. Heredia: Facultad de Filosofia y Letras, Universidad Nacional.

Mardones, J. M. (2001). Filosofía de las ciencias humanas y sociales: Materiales para una fundamentación científica. Barcelona: Anthropos.

Pavez, I. (2004). Filosofia para niños: apuesta hacia una reforma del pensamiento. Pensando desde Lipman, Morin y Accorinti. Disponible en línea: http:// antroposmodemo.com.

Restrepo, L. C. (1998). El derecho a la ternura. Bogotá: Arango Editores.

Touraine, A. (1994). Crítica de la modernidad. México D. F.: Fondo de Cultura Económica. 